\title{
On the Fracture Toughness of Ferroelectric Ceramics with Electric Field Applied Parallel to the Crack Front
}

\author{
Jianxin Wang and Chad M. Landis* \\ Department of Mechanical Engineering and Materials Science, MS 321 \\ Rice University, P.O. Box 1892 \\ Houston, TX 77251-1892 \\ Phone: (713) 348-3609, Fax: (713) 348-5423 \\ E-mail: landis@rice.edu
}

\begin{abstract}
Mode I steady crack growth is analyzed to determine the toughening due to domain switching in ferroelectric ceramics with electric field applied parallel to the crack front. A multi-axial, electromechanically coupled, incremental constitutive theory is applied to model the material behavior of the ferroelectric ceramic. The constitutive law is then implemented within the finite element method to study steady crack growth. The effects of electric field on the fracture toughness of both initially unpoled and poled materials are investigated. Results for the predicted fracture toughness, remanent strain and remanent polarization distributions, and domain switching zone shapes and sizes are presented. The effects of the plane-strain constraint are also established. Finally, the model predictions are discussed in comparison to recent experimental observations.
\end{abstract}

Keywords: fracture toughness, finite element methods, ferroelectrics, piezoelectrics, domain switching

\section{Introduction}

This work is motivated by recent experimental and theoretical investigations on the effects of electric field on the fracture toughness of ferroelectrics. The investigations presented here have focused on the specific case where the electric polarization and electric field in the sample are in the out-of-plane direction parallel to the crack front as illustrated in Figure 1. Meschke et al. [5] and Kolleck et al. [6] have observed experimentally that the steady-state fracture toughness of ferroelectrics increases with the increase of applied electric field for an initially unpolarized specimen. Additionally, Lucato et al. [7] and Hackemann and Pfeiffer [8] have observed that out-of-plane poling has practically no effect on the fracture toughness of ferroelectrics when the out-of-plane electric field is zero. Recent theoretical explanations of these phenomena have focused on the role of domain switching in the toughening process. Kolleck et al. [6] and Yang et al. [9] treat domain switching with the concepts developed for transformation toughening in partially stabilized zirconia [10,11] to analyze the fracture toughening behavior. Kreher [12] proposed a fracture model based on the balance of energy supplied by the driving forces and the total energy either dissipated by domain switching, stored in the crack wake region or consumed by the formation of new fracture surface. For these proposed theories either the details of the mechanical and electrical coupling behavior of the material is neglected or the calculation of the complicated crack tip electromechanical fields is avoided. The model to be presented in this work intends to amend these simplifications by applying a recently developed phenomenological constitutive law for ferroelectric switching within the finite element method to determine the details of the crack tip fields and the toughening due to domain switching during steady crack growth.

\section{Constitutive model}

The nonlinear constitutive response of ferroelectric materials is a result of the mechanism of domain switching. A summary of the recent developments on micro-electromechanical and phenomenological constitutive modeling of ferroelectrics can be found in Kamlah [13] and Landis [14]. The phenomenological constitutive model used for these fracture calculations is based on the work of Landis and co-workers [15-17]. This constitutive model has been verified against experimental observations and micro-electromechanical self-consistent simulations based on the model of Huber et al. [18]. Details for the formulation of the model required to investigate in-plane mechanical loading with out-of-plane electrical loading are presented in Wang and Landis [19]. Features of the constitutive response for the model material used in the fracture simulations are shown in Figure 1 below. 

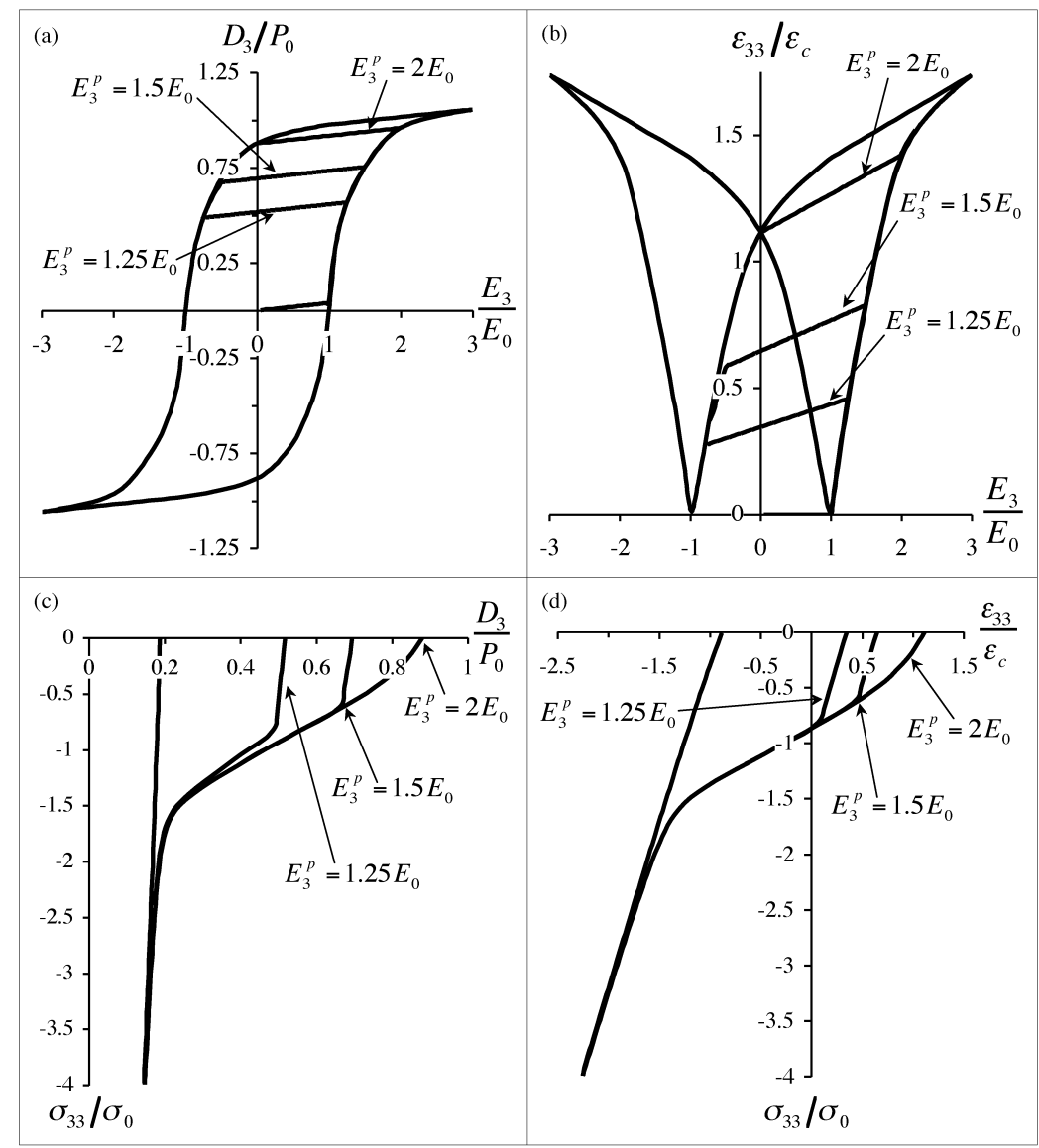

Figure 1. The uniaxial electromechanical behavior of the model material with three levels of the poling field $E_{3}^{p}$ leading to different partially poled states. (a) The electric field versus electrical displacement hysteresis loops. (b) The electric field versus strain butterfly loop. (c) The stress versus electrical displacement depolarization loop. (d) The stress versus strain loop during depolarization. Notice that the intermediate lines in (a) and (b) represent the response during the unloading of electric field, and those in (c) and (d) represent the depolarization behavior from a partially poled state.

\section{The fracture model and finite element formulation}

A schematic of the geometry and loading to be modeled here is shown in Figure 1. Two types of initial electrical states will be considered in this work: initially unpoled and initially poled states. The initially unpoled samples begin in the thermally depolarized state of the material. The initially poled samples are poled by applying a uniaxial electric field in the $x_{3}$ direction to a level of $E_{3}^{p}$ and then removing the applied field. Note that the poling field $E_{3}^{p}$ must be greater than the coercive field $E_{0}$ in order to induce remanent polarization. This entire electrical loading procedure is performed in the absence of mechanical stress. The poling process induces both residual remanent polarization and strain in the material as referenced from the thermally depolarized state. Figure 1 illustrates the (a) electric displacement versus electric field, (b) strain versus electric field response during such electrical loading, (c) depolarization due to mechanical stress, and (d) the stress versus strain response during depolarization. Due to the irreversibility of the domain switching process, there is a continuous range of partial poling states that the material can attain in the range from the initially unpoled state to a fully poled state. Note that the straight lines within the loops in Figures 1a and $1 \mathrm{~b}$ represent linear unloading during the removal of the applied electric field, and those in $1 \mathrm{c}$ and $1 \mathrm{~d}$ depict the initial behavior during depolarization by compressive stress from different partially poled states. After the initial poling step or lack of it, the electromechanical loading history for the specimen is as follows. Electric field is applied in the $x_{3}$ direction in the absence of mechanical stress. If the applied electric field is of sufficient magnitude then poling of initially unpoled samples or a reversal of poling in initially poled samples may result. In any case, this step in the electrical loading procedure induces new states of strain and electric displacement, which have been previously called the initial strain $\varepsilon_{i j}^{0}$ and initial electric displacement $D_{3}^{0}$. The initial strain and electric displacement consist of both linear reversible parts and

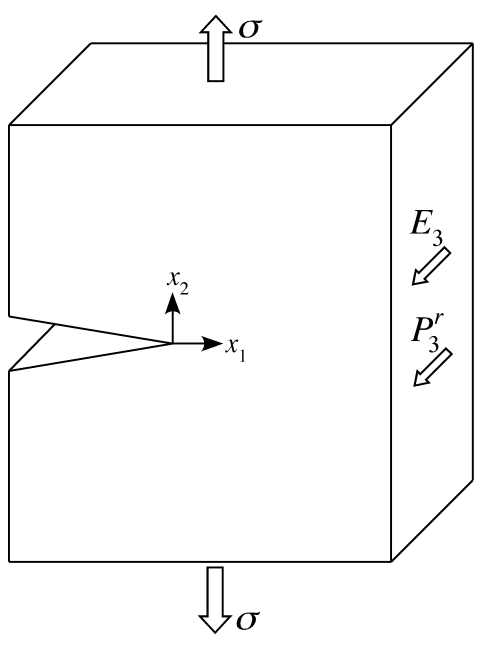

Figure 2. A schematic of the outof-plane electrical and in-plane mechanical configuration to be modeled in this work. For any given sample, the electric field and remanent polarization are aligned in $x_{3}$ direction. 
remanent parts as given by Equations (2.4) and (2.5). The final step in the loading process is to apply the in-plane mechanical loads while keeping the out-of-plane electric field fixed at the level attained in the previous step. Under plane-strain conditions the out-of-plane axial strain $\varepsilon_{33}$ is assumed to remain unchanged from its state after the electrical loading step, i.e. $\varepsilon_{33}=\varepsilon_{33}^{0}$. Steady crack growth then occurs while the in-plane mechanical loads are applied.

During crack growth, small scale switching will be assumed such that the representative height of the nonlinear switching zone near the crack tip is much smaller than any other characteristic specimen dimension such as crack length, specimen width or ligament width. Furthermore, under plane-strain conditions it is assumed that the specimen thickness is much greater than the switching zone size as well. The assumption of small scale switching will not be valid when the applied out-of-plane electric field is of sufficient magnitude to cause switching itself. However, it is assumed here that the in-plane applied mechanical loads can still be characterized by a Mode I $K$-field. Under these conditions a characteristic switching zone halfheight, $R_{s}$, can be identified as

$$
R_{s}=\frac{1}{3 \pi}\left(\frac{K_{\mathrm{I}}}{\sigma_{0}}\right)^{2}=\frac{1}{3 \pi} \frac{G E^{\prime}}{\sigma_{0}^{2}}
$$

Here $K_{\mathrm{I}}$ is the mode I stress intensity factor. Within the small scale switching approximation, the far field behavior of the solution surrounding the switching zone must asymptotically match the stresses given by the near tip elastic crack tip field. Under small scale switching conditions the prevailing mechanical conditions that govern the nonlinear behavior near the crack tip due to the geometry and far field loading of a sample are completely characterized by $K_{\mathrm{I}}$. Furthermore, the applied energy release rate, $\mathcal{G}$, is related to $K_{\mathrm{I}}$ as

$$
\mathcal{G}=\frac{K_{\mathrm{I}}^{2}}{E^{\prime}}
$$

where $E^{\prime}=E$ for plane-stress and $E^{\prime}=\frac{E}{1-v^{2}}$ for plane-strain.

The analysis presented in this paper will focus only on the toughening due to domain switching during the steady crack growth conditions described above. Within the model, crack propagation will be assumed to occur when the crack tip energy release rate $\mathcal{G}_{\text {tip }}$ reaches a critical value. In order to compute the relationship between the steady state far field applied energy release rate $\mathcal{G}_{s s}$ and $\mathcal{G}_{\text {tip }}$, a steady state finite element formulation is implemented, see Wang and Landis [19]. Under steady-state conditions, $\mathcal{G}_{\text {tip }}$ can be calculated using a formula similar to Hutchinson's $I$-integral [20] as

$$
\mathcal{G}_{\text {tip }}=I \equiv \int_{S}\left(W n_{1}-\sigma_{i j} n_{j} u_{i, 1}+D_{i} n_{i} E_{1}\right) d S
$$

where $S$ is a surface enclosing the crack tip, $n_{i}$ are the components of the unit normal directed outward from the surface, $u_{i}$ are the components of the displacement vector, $D_{i}$ are the components of the electric displacement vector, $E_{1}$ is the electric field in $x_{1}$ direction, and $W$ is the history dependent electric enthalpy density at a material point defined by

$$
W=\int_{0}^{\varepsilon_{i j}, E_{i}} \sigma_{i j} d \varepsilon_{i j}-D_{i} d E_{i}
$$

In general Equation (3.3) is a surface integral instead of a contour integral because electrical energy can enter the system from the electrodes attached to the surface of the specimen. However, due to the fact that $E_{1}=E_{2}=0$ and $E_{3}$ is constant throughout the calculation, Equation (3.3) can be simplified to a contour integral as

$$
\mathcal{G}_{\text {tip }}=\int_{\Gamma}\left(W n_{1}-\sigma_{i j} n_{j} u_{i, 1}\right) d \Gamma
$$

For a traction-free crack, the contour $\Gamma$ begins on the lower crack face, encircles the crack tip in the counterclockwise sense, and ends on the upper crack face. The calculation of $\mathcal{G}_{\text {tip }}$ is carried out after the finite element solution is obtained. 


\section{Results}

The goal of this paper is to investigate the influence of the electric field on the fracture behavior of ferroelectric materials when the electric field or the poling direction is applied parallel to the crack front. Two cases of electrical loading will be considered here, the initially unpoled and initially poled cases. After electrical loading of either case, the electric field $E_{3}$ is kept constant and the initial strain and polarization state is attained for the fracture simulation, after which the mechanical load is applied.

The primary result from each steady crack growth calculation is the ratio of the far field applied energy release rate, $\mathcal{G}_{s s}$, to the crack tip energy release rate $\mathcal{G}_{\text {tip }}$. Within the model it is assumed that crack growth occurs when $\mathcal{G}_{\text {tip }}$ reaches the intrinsic fracture toughness of the material $\mathcal{G}_{0}$. Hence the ratio $\mathcal{G}_{s s} / \mathcal{G}_{0}$ indicates the amount of toughening due to domain switching, with $\mathcal{G}_{\text {ss }} / \mathcal{G}_{0}=1$ corresponding to no toughness enhancement or R-curve behavior. With regard to R-curve behavior, $\mathcal{G}_{0}$ should be interpreted as the applied energy release rate where crack growth first begins, and $\mathcal{G}_{s s}$ is the steady state or plateau level of the applied energy release rate after sufficiently large amounts of crack growth. Figure 4 shows the ratio of $\mathcal{G}_{s s} / \mathcal{G}_{0}$ versus the applied out-of-plane electric field for a range of initial poling states. The cases associated with the solid bold curve and the dashed bold curve will be discussed first, as these

cases practically envelop the others and form an inverted butterfly loop. The solid and dashed regions of the inserted hysteresis and butterfly loops in the upper left and right hand corners correspond to the solid and dashed portions of the inverted toughness butterfly loop.

Consider a thermally depolarized material poled by a uniaxial out-of-plane electric field of magnitude $3 E_{0}$. The states of electric displacement and strain for this material can be found at the upper right corners of the hysteresis loops in Figures 1a and 1b. Then, keeping this level of applied electric field fixed, steady crack growth occurs at an applied energy release rate of $\mathcal{G}_{s s}=1.54 \mathcal{G}_{0}$.

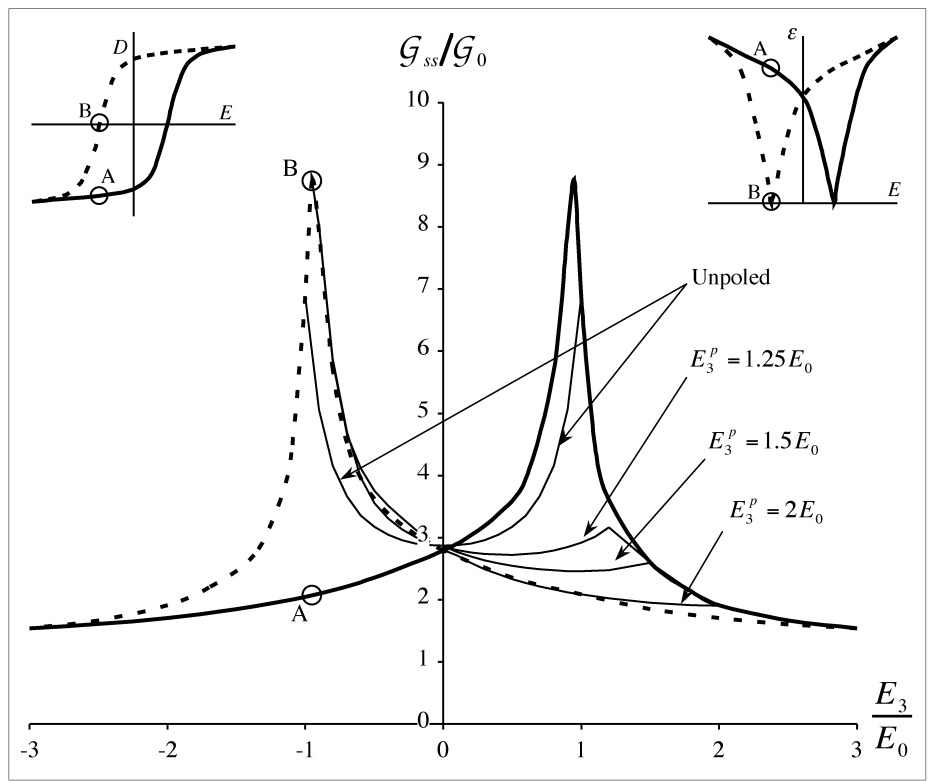

Figure 3. The normalized toughness enhancement $\mathcal{G}_{s s} / \mathcal{G}_{0}$ versus the applied out-of-plane electric field for a range of initial poling states for plane-strain conditions. The bold solid line and bold dashed line on the main plot correspond to the solid and dashed lines on the outer hysteresis and butterfly loops depicted on the inserts in the upper left and right corners. The thin lines represent the toughening behavior of unpoled or partially poled materials. Points A and B are highlighted to indicate the relationships between the electromechanical constitutive response and the fracture toughness predictions.

This level of toughening is the lowest depicted on Figure 3. However, if the applied electric field was larger, the steady state toughness would decrease even farther, approaching $\mathcal{G}_{0}$ as $E_{3} \rightarrow \infty$. The reason for this behavior is that for materials with out-of-plane remanent polarization, an applied electric field in the same direction as the polarization tends to inhibit domain switching. In other words, the alignment of the polar domains with the electric field is an energetically favorable state. Since it is the domain switching process that gives rise to the dissipation of energy and the increase in fracture toughness, any phenomenon that inhibits switching will also tend to decrease the fracture toughness. The remainder of the bold dashed curve is obtained by first poling the material with an electric field of $E_{3}^{p}=3 E_{0}$, then reversing the electric field to a lower or negative level of $E_{3}$, and finally applying the in-plane mechanical loading to produce steady crack growth. During this type of initial electrical loading the electric displacement and strain behavior of the material traces out the outermost hysteresis loops depicted in Figures 1a and $1 \mathrm{~b}$ and in the inserted plots in the upper left and right hand corners of Figure 3. As the electric field is removed, the inhibiting effect of the field on domain switching decreases and hence the fracture toughness increases. Furthermore, when the electric field is reversed sufficiently it actually enhances the driving force for domain switching and the fracture toughness of the material increases dramatically. In fact, the spikes or "butterfly legs" of the toughness versus electric field curve in Figure 3 correspond to the legs of the butterfly loops in Figure $1 \mathrm{~b}$ and the steep regions of the hysteresis loop in Figure 1a. However, if the reversal of the initial applied electric field is large enough, then the initial polarization of the material will be reversed as well, and the case where the polarization and electric field are aligned is revisited. Hence, as the initial electric field is driven to large negative levels, it will again inhibit domain switching and cause low values of the steady state fracture toughness. Finally, the bold solid curve is a mirror image of the bold dashed curve and is obtained by poling in 
the negative out-of-plane direction first. Notice that points A and B are denoted on the three loops in this figure in order to aid in the understanding of the correlation between the fundamental electromechanical constitutive behavior and the fracture toughness predictions.

One observation of the results displayed on Figure 3 that is somewhat counterintuitive is the fact that under no applied electric field the toughness enhancement of the poled samples is actually very slightly smaller than the toughness enhancement for the unpoled sample. This results is counterintuitive because one would expect that the poling process would create an out-of-plane remanent state that would allow for an increased amount of dissipation from domain switching due to in-plane mechanical loads. For example, an unpoled material loaded by an in-plane tensile stress can have a maximum inplane change in remanent strain of approximately $1.37 \varepsilon_{c}$. Whereas, a material fully poled out-of-plane can have a maximum in-plane change of remanent strain of approximately $2.06 \varepsilon_{c}$. Hence, it would appear that the poled material has a greater propensity for domain switching, dissipation and increased toughening. However, this is not the case, and the reason is due to the out-of-plane mechanical loading imposed by the

$\sigma_{33}$ stress component arising from the plane-strain constraint. As with an applied out-of-plane electric field, domains poled out-of-plane are in a low energy state with a tensile out-of-plane stress $\sigma_{33}$. If domains aligned in the out-of-plane direction are switched towards an in-plane direction, this switching process will cause a negative out-of-plane strain. In order to enforce the plane-strain constraint a tensile $\sigma_{33}$ stress will be induced by such a switching process. Therefore, the plane-strain constraint impedes domain switching in poled materials and hence the full potential toughness enhancement cannot be achieved.

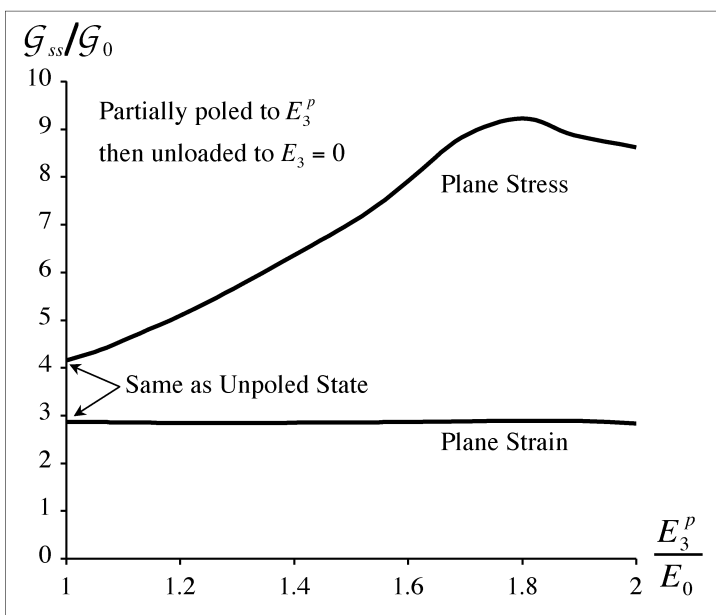

Figure 4. A comparison of the effects of plane-strain versus plane-stress out-of-plane mechanical constraint on the toughness enhancement of partially poled materials. The normalized toughness enhancement $\mathcal{G}_{s s} / \mathcal{G}_{0}$ is plotted versus the initial poling field $E_{3}^{p} / E_{0}$. For all cases the initial poling field is removed and no subsequent electric field is applied.

In order to verify and quantify the effects of the plane-strain constraint on the toughness enhancement, simulations were performed with no applied electric field on samples with differing levels of partial poling under both plane-strain and plane-stress conditions. Results for the toughness enhancement versus the level of the partial poling field are displayed on Figure 4. Note that the poling field of $E_{3}^{p}=E_{0}$ does not cause any change in remanency and so this level of poling field also corresponds to the unpoled case. Also, both of the curves in Figure 4 are practically flat for $E_{3}^{p} \geq 2 E_{0}$. From this figure it is clear that the out-of-plane mechanical constraint plays a significant role on the fracture toughness. Under plane-strain conditions the toughness behavior has a very weak dependence on the level of partial poling, while for plane-stress, i.e. $\sigma_{33}=0$, the toughness increases as the material is more fully poled. Hence, the common intuition that the toughness enhancement correlates with the potential for in-plane switching is valid for plane-stress but not for the plane-strain out-of-plane constraint.

\section{Discussion}

The model presented here differs from previous theoretical explanations of the effects of electric field and polarization on the fracture toughness of ferroelectrics in that an incremental, micro-electromechanically tested, phenomenological constitutive law has been applied instead of a discrete switching law. Additionally, in contrast to applying simplifying assumptions associated with most transformation toughening models, the details of the electromechanical fields have been obtained from finite element computations. The fields computed in this work include both the perturbing influences of ferroelectric switching and the changing piezoelectric effect that results from such switching. The detailed constitutive model applied in this work has allowed for both qualitative and quantitative characterizations of the effects of electric field on the toughening due to domain switching in ferroelectric ceramics. The model predicts a range of phenomena that indicate that the toughening is dependent on both the level of electric filed parallel to the crack front and on the polarization state. For poled materials, an electric field applied in the same direction as the polarization tends to inhibit domain switching and toughening, whereas an electric field applied opposite to the polarization directions enhances switch toughening. For initially unpoled materials, applied electric fields below the coercive field level enhance the fracture toughness of the material. As a complement to these qualitative characterizations, the quantitative predictions of the model allow for a direct comparison to some recent experimental studies.

Due to the fact that it is in contrast to conventional wisdom concerning toughening due to domain switching, the most interesting result from the model simulations is that, without an applied electric field, the toughness of an initially unpoled material is very similar to that of a material poled parallel to the crack front. For the material parameters characteristic of a soft 
PLZT material the model simulations have predicted that $\mathcal{G}_{s s}=2.87 \mathcal{G}_{0}$ for the unpoled material and $\mathcal{G}_{s s}=2.80 \mathcal{G}_{0}$ for the fully poled material. Measurements by Hackemann and Pfeiffer [8] on a soft PZT material indicate that the toughness enhancements for both poled and unpoled samples is in the range approximately $\mathcal{G}_{s s}=3 \mathcal{G}_{0}-4 \mathcal{G}_{0}$. Furthermore, Lucato et al. [7] have made similar measurements on a PZT 151 composition and found that the toughness of unpoled samples is approximately $\mathcal{G}_{s s}=2.1 \mathcal{G}_{0}$ and that of poled samples (with short-circuited electrodes on the out-of-plane surfaces) is approximately $\mathcal{G}_{s s}=1.9 \mathcal{G}_{0}$. Hence, experiments suggest that if there is any difference between the toughening of poled and unpoled samples, then the poled specimens actually incur less toughening due to domain switching than unpoled samples. The present model predicts this behavior as well. Furthermore, it was shown that the out-of-plane mechanical constraint, i.e. planestrain versus plane-stress, is the fundamental reason for this behavior.

Ultimately, the present model predicts a range of interesting effects of electric field applied parallel to the crack front on the fracture toughness, or more specifically the toughening during R-curve behavior due to domain switching during crack growth. The qualitative "shape" of the toughness enhancement versus applied electric field forms an inverted butterfly loop that correlates directly with the strain versus electric field butterfly hysteresis loop during uniaxial electrical loading. The model predicts the unintuitive behavior that the fracture toughness of a material poled out-of-plane is comparable to the toughness of an initially unpoled material. This prediction has previously been confirmed experimentally. It was demonstrated that this behavior is primarily due to the out-of-plane mechanical constraint imposed by plane strain conditions.

\section{Acknowledgement}

The authors would like to acknowledge support from the Army Research Office grant number DAAD19-02-1-0241.

\section{References}

1. Pisarenko, G.G., Chusko, V.M. and Kovalev, S. P. J. Am. Ceram. Soc., 1985, 68(5), 259.

2. Metha, K. and Virkar, A.V. J. Am. Ceram. Soc., 1990, 73(3), 567.

3. Zhang, Z. and Raj, R. J. Am. Ceram. Soc., 1995, 78(12), 3363

4. Guiu, F., Hahn, B.S., Lee, H.L., Caderon, J.M. and Reece, M.J. J. Europ. Ceram. Soc., 1997,17, 502.

5. Meschke, F., Kolleck, A. and Schneider, G.A. J. Europ. Ceram. Soc., 1997, 17, 1143.

6. Kolleck, A., Schneider, G.A. and Meschke, F.A. Acta mater., 2000, 48, 4099.

7. Lucato, S.L., Lindner, J., Lupascu, D.C. and Rodel, J. Key Eng. Mater., 2002, 206-213, 609.

8. Hackemann, S., Pfeiffer, W. J. Europ. Ceram. Soc., 2003, 23, 141.

9. Yang, W., Fang, F. and Tao, M. Int. J. Solids Struct., 2001, 38, 2203.

10. McMeeking, R.M. and Evans, A.G. J. Am. Ceram. Soc., 1982, 65, 242-246.

11. Budiansky, B., Hutchinson, J.W. and Lambropoulos, J. C. Int. J. Solids Struct., 1983, 19(4), 337.

12. Kreher, W.S. J. Mech. Phys. Solids, 2002, 50, 1029.

13. Kamlah, M. Continuum Mech. Thermodyn. 2001, 13, 219.

14. Landis, C.M. Current Opinion Solid State Mater. Sci., 2004, 8, 59.

15. Landis, C.M. J. Mech. Phys. Solids, 2002, 50, 127.

16. Landis, C.M. J. Appl. Mech., 2003, 70, 470.

17. Landis, C.M., Wang, J. and Shen, J. J. Intell. Mater. Sys. Struct., 2004, 15, 513.

18. Huber, J.E., Fleck, N.A., Landis, C.M. and McMeeking, R.M. J. Mech. Phys. Solids, 1999, 47, 1663.

19. Wang, J. and Landis, C.M. Acta mat., 2004, 52, 3435.

20. Hutchinson, J.W. Harvard University Report, DEAP S-8, Division of Applied Sciences, Harvard, 1974. 